\title{
Deriving the normalised ion-neutral collision frequency from EISCAT observations
}

\author{
J. A. Davies, M. Lester and T. R. Robinson \\ Radio and Space Plasma Physics Group, Department of Physics and Astronomy, University of Leicester, University Road, Leicester, \\ LE1 7RH, UK
}

Received: 27 November 1996 / Revised: 25 May 1997 / Accepted: 9 June 1997

\begin{abstract}
Common programme observations by the EISCAT UHF radar revealed an extended interval, post geomagnetic local noon on 03 April 1992, during which the F-region ion velocity orthogonal to the geomagnetic field was significantly enhanced, to values exceeding $2 \mathrm{~km} \mathrm{~s}^{-1}$ corresponding to a perpendicular electric field of some $100 \mathrm{mV} \mathrm{m}^{-1}$. Observations from this interval are used to illustrate a method by which estimates of the E-region ion-neutral collision frequency may be derived in the presence of enhanced electric field. From both the rotation of the ion velocity vector and the reduction in the ion velocity magnitude relative to that in the F-region, independent estimates of the normalised ion-neutral collision frequency are made at the UHF E-region tristatic altitudes; the derived values are, in general, lower than model predictions. Although initial calculations assume a stationary neutral atmosphere, first-order estimates of the E-region neutral wind are subsequently employed to calculate revised estimates of the normalised ion-neutral collision frequency; these neutral winds are derived by attributing the difference between predicted and observed enhancements in fieldparallel ion temperature to thermospheric motion. The inclusion of neutral winds, which are themselves not inconsiderable, appears to have only a limited effect on the normalised collision frequencies derived.
\end{abstract}

\section{Introduction}

Various workers have attempted to derive estimates of the ion-neutral collision frequency from observations by incoherent scatter radar. In most cases, the ion-neutral collision frequency has been determined directly from

Correspondence to: J. A. Davies the returned signal through a spectral fitting technique, or equivalently from the autocorrelation function; this technique has been used to retrieve collision frequencies from observations by the incoherent scatter radars at Arecibo (e.g. Wand and Perkins, 1968; Wand, 1976), Millstone Hill (e.g. Salah et al., 1975), Chatanika (e.g. Lathuillère et al., 1983), and the European incoherent scatter facility (EISCAT) (e.g. Flå et al., 1985; Huuskonen et al., 1986; Kirkwood, 1986; Kofman et al., 1986; Huuskonen, 1989). This regime over which the ionneutral collision frequency can be fitted successfully is limited to altitudes below some $110 \mathrm{~km}$; above this height the effect of ion-neutral collisions on the incoherent scatter spectrum, which is discussed by, e.g., Hagfors and Brokelman (1971), ceases to be significant. In addition, in fitting for the collision frequency, the ion and electron temperatures are generally assumed equal, thus restricting fittings of this type to relatively low electric field values, although Schlegel et al. (1980) successfully managed to fit simultaneously for ion temperature, electron temperature and ion-neutral collision frequency from incoherent scatter observations by the Chatanika radar.

An alternative method of determining the ion-neutral collision frequency from incoherent scatter observations was adopted by Nygrén et al. (1987). Huuskonen et al. (1984) and Nygrén et al. (1984) present expressions describing the relationship between the vertical ion velocity for various orientations of the ionospheric electric field. From these relationships, Nygrén et al. (1987) estimated the ion-neutral collision frequency from EISCAT UHF observations to altitudes approaching $140 \mathrm{~km}$. For the altitude range over which the ionneutral collision frequency could be determined from the conventional fitting technique, both methods produced comparable values which also agreed in general with estimates based on the CIRA-72 reference model atmosphere. In their method for the determination of the ion-neutral collision frequency, Nygrén et al. (1987) neglected the effect of neutral winds on ion motion in the E-region, although the authors did propose an 
approach by which their method could be amended to account for neutral flows. Indeed, in a subsequent paper, Nygrén et al. (1989) presented observations from a new EISCAT experiment, modified such that it enabled the workers to eliminate the effect of any horizontal neutral wind component in their derivation of the ion-neutral collision frequency. This modification involved the use of an eastward-tilted pointing direction of the UHF beam, in addition to the vertical observations.

In this study, a further method for calculating the ion-neutral collision frequency at E-region altitudes from the ion velocity is established; indeed this technique permits the derivation of two independent estimates of the collision frequency. The theoretical foundation of this method is outlined next.

The ion drift in the frame of reference of the neutral atmosphere, resulting from an externally imposed perpendicular electric field $\mathbf{E}$, can be expressed in the form

$\mathbf{v}_{\mathrm{i}}-\mathbf{v}_{\mathrm{n}}=\frac{1}{1+\left(v_{\mathrm{in}} / \Omega_{\mathrm{i}}\right)^{2}}\left[\frac{\mathbf{E}_{\mathrm{eff}} \times \mathbf{B}}{B^{2}}+\frac{v_{\text {in }}}{\Omega_{\mathrm{i}}} \frac{\mathbf{E}_{\mathrm{eff}}}{B}\right]$,

in which $\mathbf{v}_{\mathrm{i}}$ and $\mathbf{v}_{\mathrm{n}}$ represent the velocities of the ion and neutral populations, $v_{\text {in }}$ is the ion-neutral collision frequency, $\Omega_{\mathrm{i}}$ is the ion gyrofrequency and $\mathbf{B}$ represents the magnetic field (e.g. Schlegel and St-Maurice, 1981).

The effective electric field $\mathbf{E}_{\text {eff }}$, the electric field in the frame of reference of the thermosphere, is related to the applied electric field $\mathbf{E}$ via the expression

$\mathbf{E}_{\text {eff }}=\mathbf{E}+\mathbf{v}_{\mathrm{n}} \times \mathbf{B}$

In such a simplification, relative ion-neutral motion is restricted to the plane perpendicular to the geomagnetic field, implying an absence of effects driving field-aligned ion flow such as parallel electric fields. The effective electric field will vary with altitude, in response to any corresponding variation in the neutral wind.

An increase in the ratio of the ion-neutral collision frequency to the ion gyrofrequency, also termed the normalised ion-neutral collision frequency (Nygrén et al., 1987), characteristic of decreasing altitude, will result in the progressive rotation of the ion-neutral relative velocity vector in the field-perpendicular plane from the $\mathbf{E}_{\text {eff }} \times \mathbf{B}$ direction towards the direction of the effective electric field $\mathbf{E}_{\text {eff }}$, as discussed by, for example, Brekke et al. (1990). Furthermore, a reduction in the magnitude of the relative ion-neutral velocity is also predicted with increasing normalised ion-neutral collision frequency. With increasing ion-neutral collision frequency, the ion velocity tends towards the neutral velocity such than in the lower E-region the ion population is collisionally constrained to move with the neutral atmosphere. In the F-region, where ionneutral collisions are infrequent, the ionospheric plasma is constrained to move only along the magnetic field direction in the absence of an applied perpendicular electric field. In the presence of such a field, however, the F-region ions move, in the frame of reference of the geomagnetic field, approximately with the $\mathbf{E} \times \mathbf{B}$ drift in the field-perpendicular direction such that
$\mathbf{v}_{\mathrm{i}}=\frac{\mathbf{E} \times \mathbf{B}}{B^{2}}$

It follows from Eq. (1) that it is theoretically possible to determine the normalised ion-neutral collision frequency, at an altitude in the E-region where collisions have a significant effect on ion dynamics, independently from both the rotation of the differential ion-neutral velocity vector in the plane perpendicular to the magnetic field from the $\mathbf{E}_{\text {eff }} \times \mathbf{B}$ direction at that altitude and from the reduction in the magnitude of the field-perpendicular ion-neutral relative velocity from that of $\mathbf{E}_{\text {eff }} \times \mathbf{B}$.

The normalised ion-neutral collision frequency at a given altitude can be derived from the angle of rotation of the direction of relative ion-neutral flow at that altitude from the $\mathbf{E}_{\text {eff }} \times \mathbf{B}$ direction at that altitude, using the simple relationship

$\frac{v_{\text {in }}}{\Omega_{\mathrm{i}}}=\tan \left(\Phi\left[\mathbf{E}_{\text {eff }} \times \mathbf{B}\right]-\Phi\left[\mathbf{v}_{\mathrm{i}}-\mathbf{v}_{\mathrm{n}}\right]\right)$,

where $\Phi$ represents the angle of each vector from a reference direction in the field-perpendicular plane.

Moreover, the normalised ion-neutral collision frequency at a particular altitude can be calculated from the reduction in the magnitude of the ion-neutral relative velocity from that of $\mathbf{E}_{\text {eff }} \times \mathbf{B}$, the latter of which is expressed by the ratio $E_{\text {eff }} / B$, via the expression

$$
\left(\frac{v_{\text {in }}}{\Omega_{\mathrm{i}}}\right)^{2}=\left(\frac{\left(E_{\mathrm{eff}} / B\right)^{2}}{\left|\mathbf{v}_{\mathrm{i}}-\mathbf{v}_{\mathrm{n}}\right|^{2}}\right)-1
$$

If the neutral wind can be regarded as negligible at the altitudes where the ion-neutral collision frequency is to be determined, Eq. (1) can be simplified thus

$\mathbf{v}_{\mathrm{i}}=\frac{1}{1+\left(v_{\text {in }} / \Omega_{\mathrm{i}}\right)^{2}}\left[\frac{\mathbf{E} \times \mathbf{B}}{B^{2}}+\frac{v_{\text {in }}}{\Omega_{\mathrm{i}}} \frac{\mathbf{E}}{B}\right]$.

If the neutral atmosphere is considered stationary, it is therefore possible to determined the normalised ionneutral collision frequency at any given altitude in the Eregion from both the rotation of the ion velocity vector from the $\mathbf{E} \times \mathbf{B}$ direction at that altitude and from the reduction in the magnitude of the ion velocity from that of $\mathbf{E} \times \mathbf{B}$, the latter of which is expressed by the ratio $E / B$. Under this simplifying assumption, the solutions of equation 1 , in terms of the normalised ion-neutral collision frequency, can be expressed in the form

$\frac{v_{\text {in }}}{\Omega_{\mathrm{i}}}=\tan \left(\Phi[\mathbf{E} \times \mathbf{B}]-\Phi\left[\mathbf{v}_{\mathrm{i}}\right]\right)$

and

$\left(\frac{v_{\text {in }}}{\Omega_{\mathrm{i}}}\right)^{2}=\left(\frac{(E / B)^{2}}{v_{\mathrm{i}}^{2}}\right)-1$.

As mentioned previously, in the F-region, where ionneutral collisions are infrequent, the ions move approximately with the $\mathbf{E} \times \mathbf{B}$ drift. Thus, providing the geomagnetic field $\mathbf{B}$ can be assumed invariant with altitude between the E-region and the F-region, the Fregion ion velocity can be used in Eq. (7) and (8) to determine the normalised ion-neutral collision frequency. 
In order to calculate the ratio of the ion-neutral collision frequency to the ion gyrofrequency at a given altitude in the E-region via the above method, it is necessary to have measurements of the field-perpendicular ion vector velocity at that altitude and a corresponding measurement of the F-region drift, preferably on the same field line so that no assumptions about the spatial extent of variations in the electric field are necessary. Several versions of the field-aligned EISCAT UHF common programme 1, including CP-1-I and CP1-J, make both E-region and F-region tristatic common volume measurements and would therefore be appropriate for such a study. Ideally, simultaneous measurements of E- and F-region ion velocity are required and as this is not possible, given the nature of the UHF system, careful selection of the interval on which to test this method is necessary. Of course, this technique, in a manner similar to that of Nygrén et al. (1987) and the subsequent work of Nygrén et al. (1989), also relies on the presence of enhanced ionospheric electric fields.

The extended interval of CP-1-J in the post-noon sector of 03 April 1992, during which the F-region perpendicular ion velocity magnitude is enhanced to values approaching $2.5 \mathrm{~km} \mathrm{~s}^{-1}$, has been selected as a suitable data set on which to perform such a study. In fact, as demonstrated later, these data particularly highlight the need to account for the neutral wind in the calculation of the ion-neutral collision frequency by this method.

\section{Experimental arrangement}

In EISCAT common programme 1, version $\mathrm{J}$ (CP-1-J) the transmitter beam at Tromsø is approximately aligned along the local magnetic field direction. The remote site radars perform an 8 position scan, intersecting the transmitter beam at 6 E-region altitudes, 91, 96, 101, 109, 117 and $125 \mathrm{~km}$, and at a single F-region position, centred around $278 \mathrm{~km}$, the latter being repeated twice within each scan. The duration of each scan is $10 \mathrm{~min}$, with the dwell time in each position being approximately $60 \mathrm{~s}$. Remote site observations at the tristatic F-region altitude are, therefore, available every $5 \mathrm{~min}$, whereas for a given E-region scan position, measurements are only available every $10 \mathrm{~min}$. Four pulse schemes are transmitted: long pulse, alternating code and two power profiles. A long pulse scheme, with a pulse length of $350 \mu \mathrm{s}$, yields an altitude resolution in the local magnetic field direction of some $22 \mathrm{~km}$ extending over 21 range gates centred from approximately 140 to $600 \mathrm{~km}$ altitude. The alternating code transmission scheme is employed for high resolution Eregion observations. A 16 baud strong condition alternating code, using a baud length of $21 \mu$ s, affords an altitude resolution of approximately $3.1 \mathrm{~km}$ between 86 and $270 \mathrm{~km}$ altitude. A $21 \mu$ s power profile pulse scheme is sampled over the same range gates to enable a zero lag to be 'patched in' to the alternating code autocorrelation functions. A $40 \mu$ s power profile scheme is also included for a slightly more coarse determination of raw E- and
F-region electron density. Received signals are integrated over $5 \mathrm{~s}$.

For the present study, observations from Tromsø have been post-integrated at a temporal resolution of $5 \mathrm{~min}$ and the remote site data, over the dwell time at each scan position. The signal-to-noise ratio of the Fregion remote site and Tromsø long pulse observations employed in the present investigation are typically 20 $30 \%$ and $100-300 \%$, respectively.

\section{EISCAT CP-1-J observations of E- and F-region ion velocity}

The observing geometry of the CP-1-J experiment enables the vector ion velocity to be determined, at six E-region altitudes in addition to the F-region altitude of $278 \mathrm{~km}$, by combining the ion velocity measurements at those altitudes from each receiver site. Figure 1 illustrates both the field-perpendicular zonal (full line, positive eastward) and field-perpendicular meridional (dashed line, positive northward) components of the

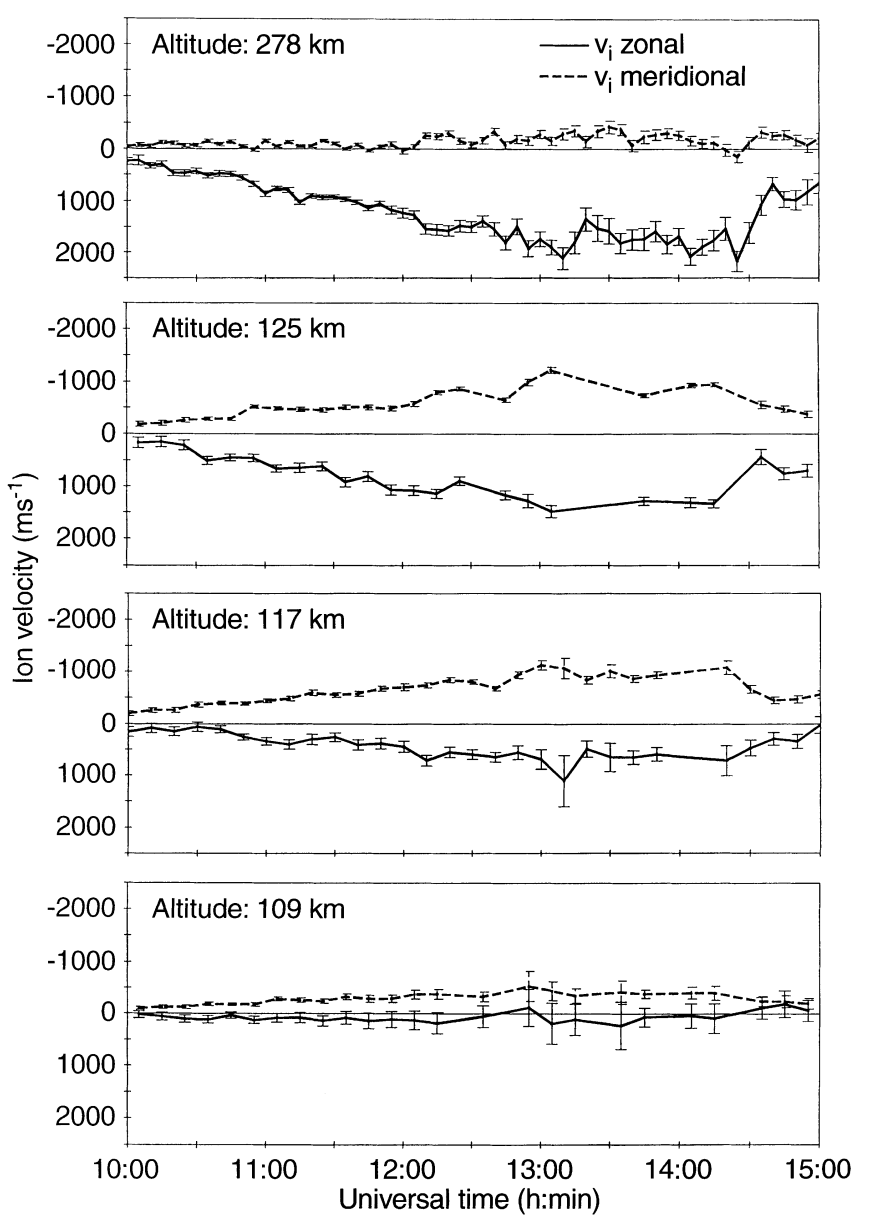

Fig. 1. Field-perpendicular zonal (full line, positive eastward) and meridional (dashed line, positive northward) ion velocity components measured by EISCAT between 10:00 and 15:00 UT on 03 April 1992. Sequential panels illustrate velocity measurements, with error estimates, at 278, 125, 117 and $109 \mathrm{~km}$ altitude 
vector ion velocity, with associated errors, at 278, 125, 117 and $109 \mathrm{~km}$ altitude between 10:00 and 15:00 UT on 03 April 1992. The quoted errors include uncertainties due to the analysis and the random error (du Castel and Vasseur, 1972). Ion velocity components are presented only where the signal-to-noise measurement at each of the three UHF receiver sites employed in their calculation exceeds a threshold of $2 \%$. Measurements of the field-orthogonal ion velocity from the three lower Eregion tristatic altitudes, 91, 96 and $101 \mathrm{~km}$, are not presented as many of the incoherent scatter signals returned from those altitudes were not sufficiently well determined for analysis. Moreover, many of those from which an ion velocity estimate could be derived were of low signal-to-noise ratio, a consequence of the low electron density at these altitudes during this interval. The ion velocity components calculated at $278 \mathrm{~km}$ altitude were derived from long pulse transmission. At the E-region tristatic altitudes, the velocities were derived from a combination of long pulse observations from the remote sites and alternating codes at Tromsø.

At the F-region tristatic altitude of $278 \mathrm{~km}$, the fieldperpendicular zonal component of the ion velocity increases to a maximum approaching $2500 \mathrm{~m} \mathrm{~s}^{-1}$ in a westward direction whereas the meridional component remains comparatively low throughout the interval, less than $400 \mathrm{~m} \mathrm{~s}^{-1}$. The zonal component of the ion velocity remains consistently westward at $278 \mathrm{~km}$ altitude, whereas the meridional ion velocity, although predominantly directed northward, undergoes several reversals in direction. At $125 \mathrm{~km}$ altitude, the magnitude of the zonal component of the ion velocity is reduced with respect to that at $278 \mathrm{~km}$, attaining a maximum value of around $1500 \mathrm{~m} \mathrm{~s}^{-1}$ between 13:00 and 14:30 UT. Conversely, the field-perpendicular meridional ion velocity component at $125 \mathrm{~km}$ altitude greatly exceeds that at the F-region tristatic altitude and remains consistently northward throughout the interval. Whereas at $278 \mathrm{~km}$ altitude the field-perpendicular zonal component of the ion velocity greatly exceeds the corresponding meridional component, at $125 \mathrm{~km}$ altitude the meridional ion velocity component is not significantly less than the zonal velocity component. At $117 \mathrm{~km}$, the zonal ion velocity component is generally lower than that observed at $125 \mathrm{~km}$ whereas the magnitude of the meridional component is comparable at these two Eregion intersection altitudes. In contrast to the behaviour observed at higher altitudes, at $117 \mathrm{~km}$ the magnitude of the meridional component of the ion velocity exceeds that of the zonal component over much of the interval. At $109 \mathrm{~km}$ altitude, neither of the fieldperpendicular components of the ion velocity attain values in excess of $500 \mathrm{~m} \mathrm{~s}^{-1}$, although the meridional ion velocity is significantly greater than the corresponding zonal component. The zonal ion velocity component at $109 \mathrm{~km}$ does not remain consistently westward as is observed at higher altitudes; there is evidence for the occurrence of short-lived instances of eastward zonal flows just before 13:00 UT and between 14:30 and 15:00 UT, although the estimated errors on the velocity measurements at this altitude are large.
The behaviour of the individual field-perpendicular ion velocity components implies both a progressive rotation in the orientation of the ion velocity vector in the field-orthogonal plane, from zonal to meridional, and a reduction in the velocity magnitude with decreasing altitude, effects which are more clearly demonstrated in Fig. 2. Figure 2 presents the field-orthogonal ion velocity magnitude (upper panel) and the angle from the meridional northward direction of the field-orthogonal ion velocity vector (lower panel) with associated error estimates, at the altitudes of 278, 125, 117 and $109 \mathrm{~km}$ over the same time interval as Fig. 1. In the lower panel of Fig. 2, positive angles indicate eastward zonal ion velocities and negative angles represent westward directed zonal flow.

At $278 \mathrm{~km}$ altitude, the ion velocity vector is directed at an angle of between 10 and $20^{\circ}$ to the field-orthogonal westward direction, and its magnitude increases from an initial value of $250 \mathrm{~m} \mathrm{~s}^{-1}$ at 10:00 UT to approximately $2250 \mathrm{~m} \mathrm{~s}^{-1}$. Short periods during which meridional flow
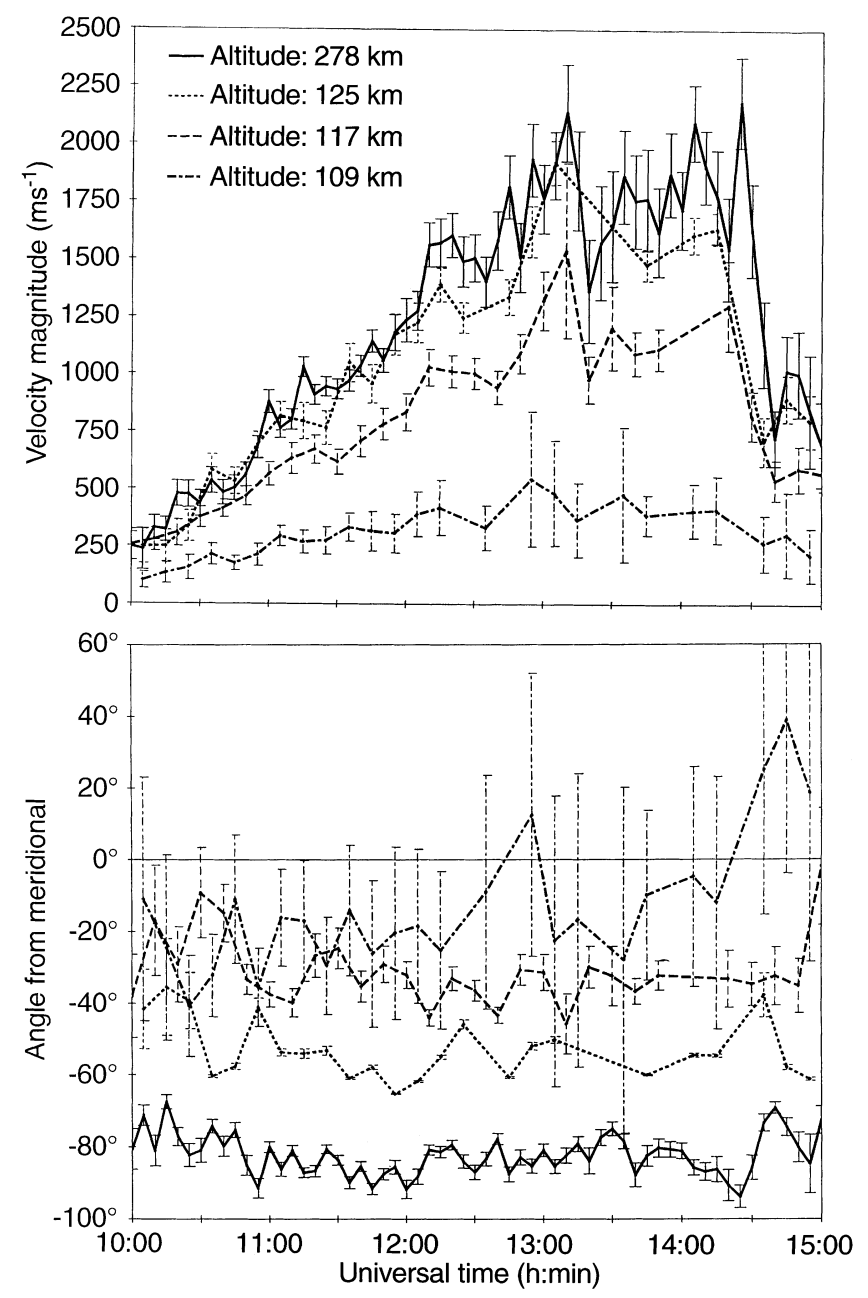

Fig. 2. Magnitude (upper panel) and direction (lower panel) of the field-perpendicular ion vector velocity derived from EISCAT measurements at 278,125, 117 and $109 \mathrm{~km}$ altitude, from 10:00 to 15:00 UT on 03 April 1992. Directions are represented by the angle of ion flow from meridional northward, positive indicating an eastward zonal flow, and observations from each altitude are differentiated by their line style 
at the F-region tristatic altitude was southward (see Fig. 1) are represented by angles of less than $-90^{\circ}$, for example $-100^{\circ}$ just prior to $14: 30 \mathrm{UT}$. At an altitude of $125 \mathrm{~km}$ the field-orthogonal ion velocity magnitude is slightly reduced with respect to that at $278 \mathrm{~km}$ over much of the $5 \mathrm{~h}$ interval, typically by some $10 \%$. The ion velocity vector, however, has been significantly rotated towards the meridional northward direction, by an angle approaching $30^{\circ}$. The magnitude of the ion velocity at $117 \mathrm{~km}$ altitude attains a maximum of approximately $1500 \mathrm{~m} \mathrm{~s}^{-1}$ at 13:00 UT; the velocity vector is rotated from that at an altitude of $125 \mathrm{~km}$ by about $20^{\circ}$. At $109 \mathrm{~km}$ altitude the orthogonal ion velocity magnitude is reduced to less than one fifth of its value at the F-region tristatic altitude and is rotated by some $60^{\circ}$ from the direction of the F-region ion velocity.

Both the rotation in the field-perpendicular ion velocity observed by EISCAT during the interval mentioned, from a near geomagnetic zonal westward direction towards the meridional north with decreasing altitude and the reduction in the magnitude of the ion velocity from that measured at F-region altitudes, illustrate the effect of increasing collisional coupling with the neutral atmosphere on the motion of the ion population. Indeed, the sense of rotation of the ion velocity is consistent with what would be anticipated given the direction of the F-region flows; westward F-region ion flows indicate a northward applied electric field.

The altitude variation of ion velocity in the auroral ionosphere was previously observed, and discussed in detail, by Brekke et al. (1990) in their study of E-region currents. The authors examined observations from an earlier version of EISCAT common programme 1, CP$1-\mathrm{H}$, in which tristatic measurements were made at four E-region altitudes $(101,109,119$ and $132 \mathrm{~km})$ and in the F-region. It must be realised, as also noted by Brekke et al. (1990), that in the presence of an applied electric field, a progressive rotation in the observed perpendicular ion velocity and reduction in the velocity magnitude with decreasing altitude will not necessarily be observed. With increasing ion-neutral collision frequency there is certainly a progressive rotation of the relative ionneutral velocity vector from the direction of $\mathbf{E}_{\mathrm{eff}} \times \mathbf{B}$ to that of $\mathbf{E}_{\text {eff }}$, but this does not necessarily imply that the ion velocity will be observed to rotate progressively, as the effective electric field, or more specifically the neutral wind, varies with altitude. Similarly, the ion velocity magnitude will not necessarily be observed to reduce progressively with decreasing altitude.

\section{Derivation of the normalised ion-neutral collision frequency}

The ratio of the ion-neutral collision frequency to the ion gyrofrequency, the normalised ion-neutral collision frequency, has been derived at the three highest CP-1-J E-region intersection altitudes, 109,117 and $125 \mathrm{~km}$ altitude during the interval of enhanced electric field in the post-noon sector on 03 April 1995. In the initial calculations of the normalised collision frequency the neutral wind in the E-region is assumed to be negligible, thus expressions (7) and (8) are employed. The validity of this assumption is discussed later, particularly with reference to the present observations. Independent estimates of the normalised ion-neutral collision frequency during this interval have been derived from the rotation of the observed ion velocity vector at each of the E-region altitudes from the direction of the observed F-region ion velocity at $278 \mathrm{~km}$, representative of that of $\mathbf{E} \times \mathbf{B}$, and from the reduction in the velocity magnitude from that observed at $278 \mathrm{~km}$ altitude, the latter is expressed by the ratio $E / B$. As mentioned previously, in assuming that the F-region ion velocity measurements are representative of $\mathbf{E} \times \mathbf{B}$ in the $\mathrm{E}$ region, the geomagnetic field must be assumed to be approximately equal in both its magnitude and direction between the F-region tristatic volume and the E-region altitudes of interest. Calculations of the IGRF model of the geomagnetic field at the position of EISCAT indicate that between the F-region and the E-region the magnitude and direction of the magnetic field vary by less than $5 \%$ and $1^{\circ}$, respectively. The resultant effect on the derived value of the normalised ion-neutral collision frequencies would be less than 5\%, comparable, indeed, to the uncertainities in the EISCAT measurements.

Figure 3 presents time series of normalised ionneutral collision frequency, with associated errors, at $125 \mathrm{~km}$ (upper panel), $117 \mathrm{~km}$ (central panel) and $109 \mathrm{~km}$ altitude (lower panel) from 10:00 to 15:00 UT on 03 April 1992, derived from the EISCAT CP-1-J observations of the rotation of the ion velocity vector (full line) and the reduction in the velocity magnitude (dashed line) from those measured at the F-region altitude of $278 \mathrm{~km}$. Additionally, modelled values of the normalised collision frequency at each of these altitudes are illustrated by a dotted line. The normalised ionneutral collision frequency is modelled at the CP-1-J tristatic E-region altitudes using the MSIS-86 model thermosphere (Hedin, 1987) and the IGRF model, appropriate to the above date and the geographic location of EISCAT. The ion-neutral collision frequency is modelled for an ion population comprising $\mathrm{NO}^{+}$and a neutral atmosphere consisting of $\mathrm{O}_{2}, \mathrm{~N}_{2}$ and $\mathrm{O}$, with the neutral densities being taken from the MSIS-86 atmospheric model. The collisions of $\mathrm{NO}^{+}$with all aforementioned neutral species are non-resonant interactions, combining long-range polarisation attraction and short-range repulsion, and, unlike resonant charge exchange interactions in which an ion collides with its parent neutral, such polarisation type collisions are not dependent on the effective temperature which varies with electric field. The ion-neutral collision frequency is calculated using tabulated values of the collision coefficients cited in the review of Schunk and Nagy (1980) such that

$$
\begin{aligned}
v_{\text {in }}= & 4.34 \times 10^{-16} \mathrm{~N}_{\left[\mathrm{N}_{2}\right]}+4.28 \\
& \times 10^{-16} \mathrm{~N}_{\left[\mathrm{O}_{2}\right]}+2.44 \times 10^{-16} \mathrm{~N}_{[\mathrm{O}]}
\end{aligned}
$$



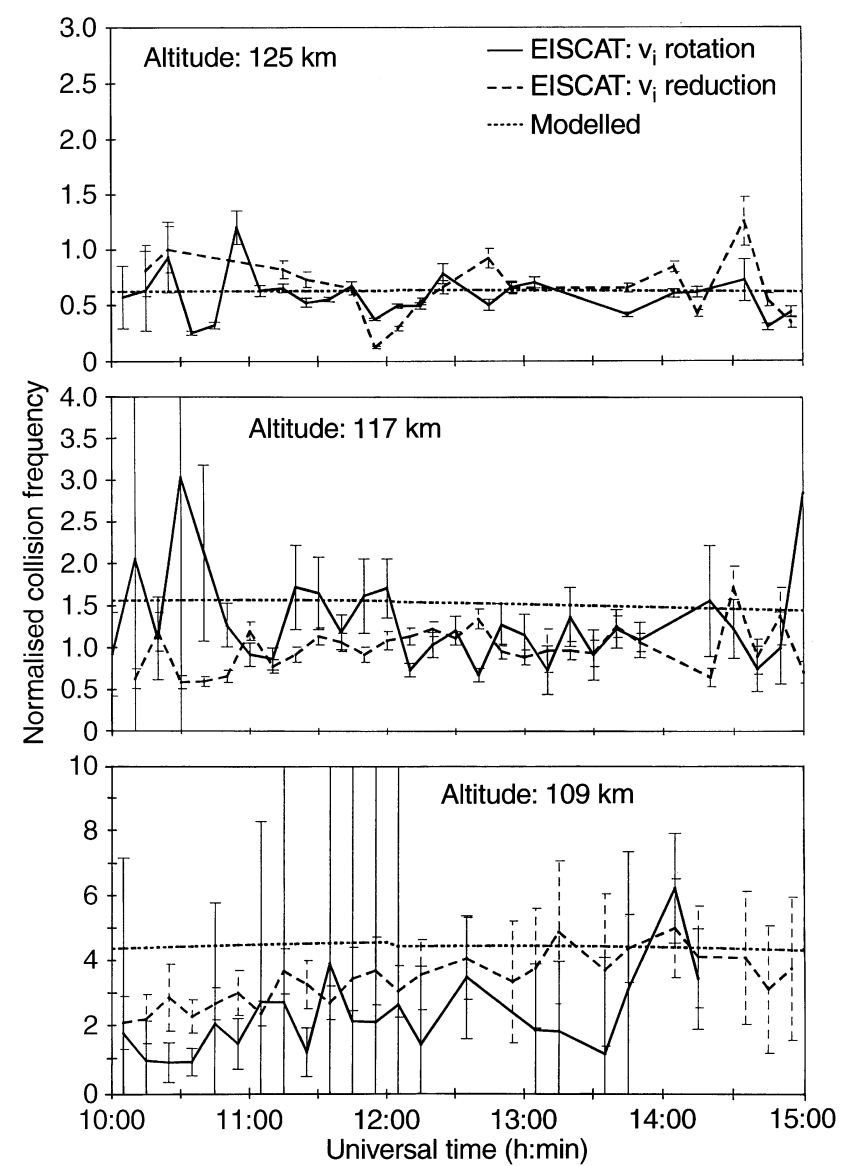

Fig. 3. Normalised ion-neutral collision frequency derived from EISCAT observations of ion velocity rotation (full line) and ion velocity magnitude reduction (dashed line) at 125 (upper panel), 117 (middle panel) and $109 \mathrm{~km}$ altitude (lower panel), from 10:00 to 15:00 UT on 03 April 1992. Modelled values are also illustrated, represented by a dotted line

where the collision coefficients are quoted in units of $\mathrm{m}^{3} \mathrm{~s}^{-1}$ (Schunk and Walker, 1973). The ion gyrofrequency is calculated, again for $\mathrm{NO}^{+}$ions, using magnetic field estimates from the IGRF model of the geomagnetic field.

At E-region altitudes, analysis of EISCAT autocorrelation functions assumes a totally molecular ion population with a mean relative mass of 30.5 , equivalent to an ion composition comprising $75 \% \mathrm{NO}^{+}$and $25 \%$ $\mathrm{O}_{2}^{+}$, although in modelling the normalised ion-neutral collision frequency an ion gas comprising totally $\mathrm{NO}^{+}$is assumed. However, even in the extreme case where $100 \% \mathrm{O}_{2}^{+}$is assumed, the modelled collision to gyrofrequency ratios are almost identical to those calculated assuming solely $\mathrm{NO}^{+}$. The variation with time of the modelled values of normalised ion-neutral collision frequency over the five hour interval, albeit small, results primarily from the variation with universal time of the densities of the neutral species yielded by the MSIS-86 model. The discontinuity in the modelled values at 12:00 UT is a consequence of the parameterisation of MSIS- 86 by $A_{p}$, a three hourly magnetic index.

At $125 \mathrm{~km}$ altitude, the values of normalised collision frequency derived from the EISCAT observations of both ion velocity rotation and reduction in velocity magnitude are similar, although rather variable, and are comparable to those predicted. At the tristatic altitude of $117 \mathrm{~km}$, the derived values of the normalised ionneutral collision frequency remain comparable, but are generally somewhat less than the modelled values. At $109 \mathrm{~km}$ altitude, the tendency for the modelled ratio to exceed those derived from the EISCAT observations continues, but, it is noticeable that the value of the normalised collision frequency derived from the rotation of the ion velocity (lower panel of Fig. 3, solid line) is, in general, significantly less than that derived from the observations of the reduction of the ion velocity magnitude (lower panel of Fig. 3, dotted line). Furthermore, at this altitude, the derived values of normalised collision frequency demonstrate a gradual increase with time through the interval; this feature is discussed in more detail later on in the work. At altitudes below $109 \mathrm{~km}$ the modelled values of normalised collision frequency (not shown) tend also to exceed those derived from the EISCAT measurements, although the latter are extremely variable. The altitude at which the modelled normalised collision frequency is of value unity is approximately $119 \mathrm{~km}$, compared with $117 \mathrm{~km}$ for the ratio derived from the EISCAT ion velocity observations. The mean and standard deviation of normalised collision frequency derived from EISCAT observations of ion velocity rotation and reduction, and modelled using the MSIS-86 and IGRF models, are given in Table 1.

Larger electric fields enable more accurate determination of the normalised ion-neutral collision frequency; the mean values and associated standard deviations of the normalised ion-neutral collision frequencies quoted in Table 1 are therefore calculated from those estimates of normalised collision frequency derived between 11:00 and 14:30 UT, corresponding to an electric field exceeding $35 \mathrm{mV} \mathrm{m}^{-1}$ in magnitude. Moreover, it should be noted that the rotation in the ion velocity vector is likely to provide a more accurate estimate of the normalised ion-neutral collision frequency at higher altitudes in the E-region, as changes in the orientation of the velocity vector with normalised collision frequency are more pronounced for small values of the latter. At $125 \mathrm{~km}$ altitude, the ion velocity magnitude is comparable to that in the F-region, indeed sometimes it exceeds that at $278 \mathrm{~km}$ altitude in which case an estimate of the collision frequency is not possible, whereas the velocity vector is significantly rotated between these two alti-

Table 1. Mean normalised ion-neutral collision frequencies derived from EISCAT observations taken between 11:00 and 14:30 UT. Standard deviations are also given

Normalised ion-neutral collision frequency

\begin{tabular}{lllllll}
\hline Altitude $(\mathrm{km})$ & \multicolumn{2}{c}{ Velocity rotation } & \multicolumn{2}{c}{ Velocity reduction } & \multicolumn{2}{c}{ Modelled } \\
\hline 125 & 0.6 & 0.1 & 0.6 & 0.3 & 0.63 & 0.01 \\
117 & 1.2 & 0.5 & 1.1 & 0.2 & 1.53 & 0.03 \\
109 & 2.7 & 1.2 & 3.7 & 0.7 & 4.49 & 0.06 \\
\hline
\end{tabular}


tudes (see Fig. 2). The normalised ion-neutral collision frequency could, therefore, theoretically be derived from the rotation in the ion velocity at higher altitudes than $125 \mathrm{~km}$ given measurements of the ion velocity at the appropriate altitudes; for a normalised ion-neutral collision frequency of 0.2 , appropriate to an altitude approaching $140 \mathrm{~km}$, the ion velocity vector would still be rotated by some $10^{\circ}$. Conversely, at lower altitudes the reduction in the magnitude of the ion velocity is more pronounced; the ion velocity magnitude exhibits a significant reduction between 117 and $109 \mathrm{~km}$ altitude, from a maximum exceeding $1500 \mathrm{~m} \mathrm{~s}^{-1}$ to less than $500 \mathrm{~m} \mathrm{~s}^{-1}$.

Various previous authors have compared incoherent scatter measurements of the ion-neutral collision frequency with those predicted from atmospheric models. Measurements of the collision frequency from the Arecibo radar, presented by Wand and Perkins (1968), compared favourably with those from the CIRA-65 model thermosphere. Similarly, both Wickwar et al. (1981) and Lathuillère et al. (1983) found that measurements of the collision frequency from the Chatanika incoherent scatter radar were consistent with values derived from the JACCHIA-71 model. Measurements by the Chatanika radar (Schlegel et al., 1980), however, yielded collision frequencies noticeably less than those calculated from the CIRA-72 atmospheric model (StMaurice et al., 1981), a result which the authors attributed to an overestimate in the model densities. Huuskonen et al. (1986) found EISCAT measurements of ion-neutral collision frequency to be in fairly good agreement with CIRA-72, whereas a study by Kirkwood (1986) revealed collision frequency measurements which, in general, significantly exceeded predictions from both CIRA-72 and the MSIS-83 thermospheric model; the results of Kirkwood (1986), based on an extensive set of EISCAT observations, were subsequently used in the development of MSIS-86. Later work by Huuskonen (1989), also with the EISCAT radar, showed that while agreement with MSIS-86 was found in July, in February and August the observed collision frequencies were greater than the model values. In all of the studies mentioned the collision frequencies were retrieved through a fitting technique and consequently limited to altitudes below about $110 \mathrm{~km}$. The velocity method of Nygrén et al. (1987), which enabled the ion-neutral collision frequency to be successfully determined to altitudes approaching $140 \mathrm{~km}$, yielded collision frequencies which, although in general agreement with model results from CIRA-72, tended to be less than the model values at altitudes above some $110 \mathrm{~km}$. Subsequent comparison of these results with MSIS-86, by Huuskonen (1988), however, faired better at these altitudes. The work by Nygrén et al. (1989), in which the authors modified the method of Nygrén et al. (1987) to eliminate the effect of horizontal atmospheric motion, yielded ionneutral collision frequencies which were consistently greater than those predicted using the MSIS- 86 model.

Nygrén (1996) reviews much of the previous work regarding the study of ion-neutral collision frequency in the E-region using the EISCAT radar, including that of
Flå et al. (1985), Huuskonen et al. (1986), Kirkwood (1986), Kofman et al. (1986), Nygrén et al. (1987, 1989) and Huuskonen (1989). In particular, the author compares selected profiles of ion-neutral frequency from all of these papers with values derived from the MSIS-86 model, a comparison which reveals that, in general, previous measurements of ion-neutral collision frequency, determined both by fitting and from the ion velocity, tend to exceed values derived from the MSIS-86 thermospheric model.

In contrast to much of the previous work, the values of the ion-neutral collision frequency derived in the present study tend to be lower than those which would be expected in a neutral atmosphere described by the MSIS-86 model. However, a rigorous comparison of the present results with previous measurements of ionneutral collision frequency is not included in this work. Rather, the intention is to demonstrate an alternative method to that of Nygrén et al. (1987) by which the normalised ion-neutral collision frequency can be derived from EISCAT observations of ion velocity under conditions of enhanced electric fields.

It is, at this juncture, informative to point out the relative merits and disadvantages of this method over that of Nygrén et al. (1987). These authors employed previously derived functional relationships between the electric field and the vertical ion velocity to derive the ion-neutral collision frequency during an EISCAT UHF special programme run, in which the transmitter was pointed vertically and the remote sites remained at a single F-region tristatic volume. One distinct advantage of their method is that simultaneous electric field and Eregion vertical ion velocity measurements were thus available (albeit not on the same field line), whereas, for the present method, the remote site radars must scan between the F-region and the E-region intersection volume of interest and over this time, of order several minutes, the ionosphere must be assumed static. Thus, the present method is inappropriate under situations of rapidly varying electric fields associated with substorm activity as observed by, for example, Williams et al. (1990). This factor significantly influenced the selection of an interval on which to perform this study; the postnoon sector of 03 April 1992 was chosen not only due to the presence of a substantial electric field but also because that field appeared to remain relatively constant over a long time scale, much longer than the several minute gap between E- and F-region tristatic observations.

Furthermore, the method of Nygrén et al. (1987) generates height profiles of collision frequency whereas the present method enables the collision frequency to be determined only at the E-region tristatic altitudes. The more coarse altitude resolution which is, therefore, available to the present authors is not, however, a serious disadvantage as the ion-neutral collision frequency is believed to vary smoothly with height.

Although CP-1-K tristatic observations are available below $109 \mathrm{~km}$, their use has not been possible in the present study due to the low signal-to-noise ratio, particularly of measurements from the remote site 
receiver at Sodankylä which, due to the geometry of the experiment, is far from the intersection volume. It is not clear that, even given satisfactory velocity measurements, it would be easy to obtain good estimates of the collision frequency must below $109 \mathrm{~km}$ with this method, since neither the direction or the magnitude of the ion velocity would be expected to vary strongly with altitude. At these altitudes it is, however, possible to derive the ion-neutral collision frequency from a conventional fitting method, although, as stated in the introduction, the assumption of equal ion and electron temperatures is somewhat compromised under situations of large electric fields.

The major advantage of the technique documented in this study is that it enables two simultaneous estimates of the collision frequency to be derived for any estimate of the E-region ion velocity. Both this method, and that of Nygrén et al. (1987), enable the collision frequency to be derived to much higher altitudes than by conventional fitting, although in the present study this is limited to $125 \mathrm{~km}$ by the geometry of the experiment.

\section{Estimation of the neutral wind}

Recently, a number of authors have attempted to derive estimates of the E-region neutral wind from EISCAT observations of ion velocity (e.g. Virdi et al., 1986; Williams and Virdi, 1989; Kunitake and Schlegel, 1991; Virdi and Williams, 1993; Brekke et al., 1994). These studies have mainly been in relation to the investigation of tidal oscillations in the lower thermosphere, and were thus restricted to relatively undisturbed conditions. In most of these studies the authors assumed, following the work of Brekke et al. (1973), that, during such quiet conditions, the ion motion in the E-region was principally governed by that of the neutral atmosphere such that measurements of the ion velocity could be used as a monitor of neutral velocities provided a small correction for the effect of the electric field was introduced. The approach adopted by these authors is essentially the antithesis of that adopted in the present study; the correction employed to negate the effect of electric fields invariably assumed modelled values of the normalised ion-neutral collision frequency. In the present study, first-order estimates of the E-region neutral wind are derived separately, from a solution of the simplified ion energy balance equation.

The ion energy balance equation may be significantly simplified on application to a realistic ionosphere in the presence of large electric fields and, if solved with respect to ion temperature appropriate to the field-parallel ion population, can be expressed in the form

$T_{\mathrm{i} \|}-T_{\mathrm{eq}}=\frac{\left\langle m_{n}\right\rangle}{2 k_{\mathrm{B}}} \beta_{\|}\left(\mathbf{v}_{\mathrm{i}}-\mathbf{v}_{\mathrm{n}}\right)^{2}$

in which $T_{\mathrm{i} \|}$ is the field-parallel ion temperature, $k_{\mathrm{B}}$ is the Boltzmann constant and $\left\langle m_{n}\right\rangle$ and $\beta_{\|}$represent the mean neutral mass and field-parallel ion temperature partition coefficient, respectively (e.g. St-Maurice and Schunk, 1977).
The ambient, or equilibrium ion temperature, $T_{\mathrm{eq}}$, defined as the ion temperature in the absence of strong frictional heating, is equivalent to the sum of the neutral temperature and a small contribution to the ion temperature due to electron-ion thermal energy exchange. The term on the left hand side, thus represents the steady-state enhancement in the field-parallel ion temperature in response to ion frictional heating; such steady-state conditions are attained in a characteristic time which is the inverse of the ion-neutral collision frequency.

Predictions of the enhancement in the field-parallel ion temperature at the three E-region tristatic altitudes of 109,117 and $125 \mathrm{~km}$ altitude were derived from the observed ion velocity magnitudes at those altitudes from the simplified form of the ion energy balance equation given above, assuming a stationary thermosphere. The average neutral mass, $\left\langle m_{n}\right\rangle$, (e.g. Schunk and Walker, 1972) has been calculated for an ion population comprising $\mathrm{NO}^{+}$and a neutral population of $\mathrm{O}_{2}, \mathrm{~N}_{2}$ and $\mathrm{O}$, neutral densities being taken from MSIS-86 thermospheric model. The expressions for the collision frequencies of the polarisation interactions between these ion and neutral species are those cited in the review of Schunk and Nagy (1980). The field-parallel ion temperature partition coefficient, $\beta_{\|}$, is evaluated employing the expression as quoted by, for example, Løvhaug and Flå (1986) and McCrea et al. (1993), again appropriate to the molecular ion species $\mathrm{NO}^{+}$. In the calculation of the parallel temperature partition coefficient, it is necessary to adopt a value for $\alpha_{\text {in }}$, a numerical constant which is the ratio of generalised transport cross sections involved in the momentum transfer collision frequency. StMaurice and Schunk (1977) presents values of $\alpha_{\text {in }}$ for various collision mechanisms and ranges of energy. The value adopted for $\alpha_{\text {in }}$ is taken from Table 1 of StMaurice and Schunk (1977) for polarisation collisions of type B; this value was adopted by those authors for all non-resonant ion-neutral interactions. Values of $\beta_{\|}$ estimated from this equation, with neutral densities, again taken from MSIS-86, range between some 0.53 and 0.55 which are comparable with theoretical values derived by St-Maurice and Schunk (1977) for $\mathrm{NO}^{+}-\mathrm{N}_{2}$ interactions and estimates from EISCAT observations by Lathuillère et al. (1991).

The field-parallel ion temperature enhancement predicted from CP-1-J measurements of the ion velocity at $109,117,125 \mathrm{~km}$ altitude between 10:00 and 15:00 on 03 April 1992 are compared to those observed. To define an observed enhancement in the parallel ion temperature it is necessary to define an unperturbed ion temperature at each altitude. Ion temperature exhibits only a slight diurnal variation, and the modal value over an extended quiet interval is an adequate representation of its unperturbed value (McCrea et al., 1991). Therefore, the unperturbed ion temperature is defined as the modal parallel ion temperature, calculated from its distribution over the 03 April 1992. The problems inherent in the derivation of ion temperatures by analysis of the returned incoherent scatter signal during intervals of high ion flow velocities have been discussed extensively 
elsewhere (e.g. McCrea et al., 1995; Davies et al., 1995). In the direction parallel to the magnetic field, uncertainties in the derived ion temperature principally result from the adoption of a time invariant ion composition model. An increase in the proportion of molecular ions above that assumed in the analysis can result in the significant underestimate in the values of ion temperature yielded by the analysis. The ion composition profile adopted for standard EISCAT analysis, however, already assumes an ion population totally comprised of molecular species below an altitude of $150 \mathrm{~km}$.

Figure 4 presents time series of the observed (full line) and expected (dashed line) field-parallel ion temperature enhancement at 125, 117 and 109 altitude from 10:00 to 15:00 UT on 03 April 1992. The maximum observed enhancement in the field-parallel ion temperature decreases from approximately $2000 \mathrm{~K}$ at $125 \mathrm{~km}$ to $200 \mathrm{~K}$ at $109 \mathrm{~km}$ altitude, a consequence of the reduction in the ion-neutral velocity in response to increased collisional coupling between the two populations. At all three altitudes under consideration the enhancement predicted by Eq. (10) generally exceeds that observed, by as much as $1000 \mathrm{~K}$. This could be a consequence of assuming that the neutral velocity was

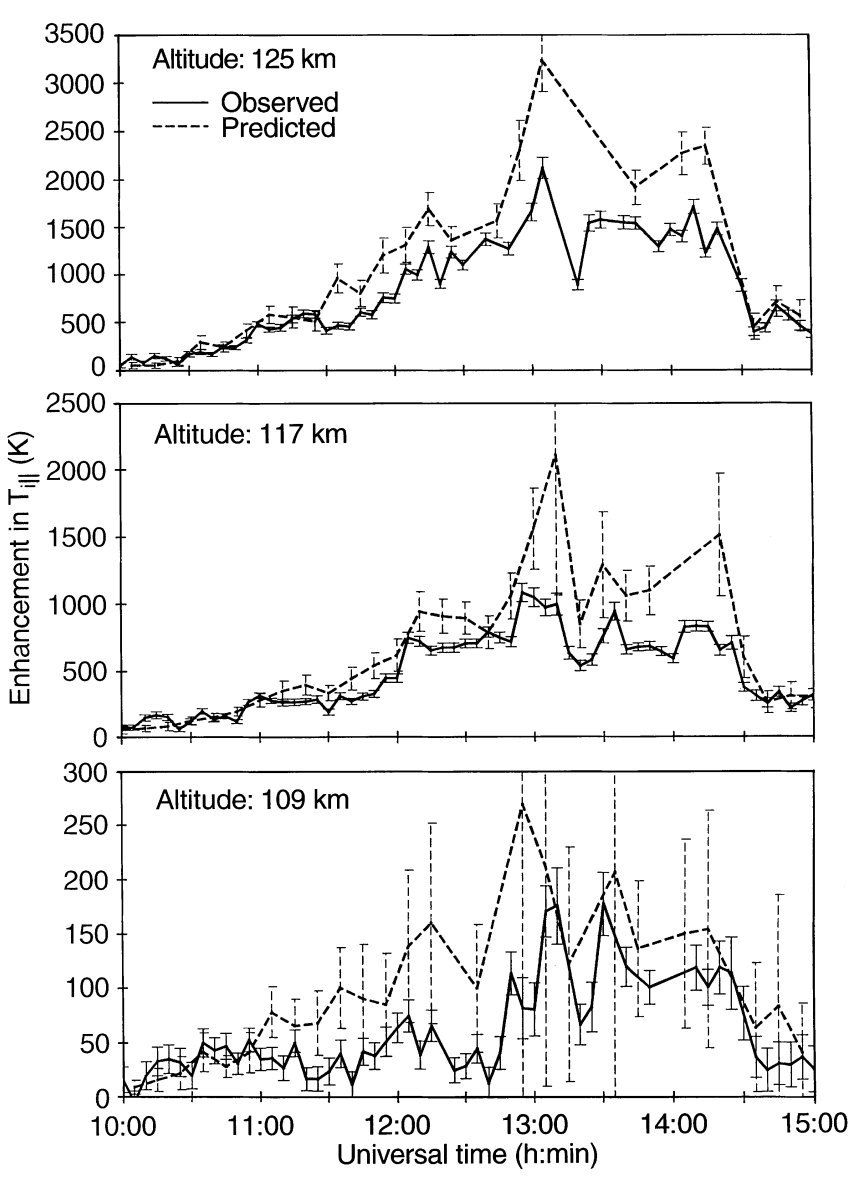

Fig. 4. Observed (full line) and modelled (dashed line) field-parallel ion temperature enhancement at 125 (upper panel), 117 (middle panel) and $109 \mathrm{~km}$ altitude (lower panel), from 10:00 to 15:00 UT on 03 April 1992 negligible when predicting the field-parallel ion temperature enhancement. The existence of a neutral wind due to ion drag would reduce the ion-neutral velocity difference, thus suppressing ion frictional heating, especially over such an extended period of enhanced ion flow. An additional mechanism has been suggested to heat the ions at E-region altitudes. McBride et al. (1972) argue that turbulent heating of the E-region ions by the modified two-stream instability will occur during intervals of large electric field in a process analogous to electron turbulent heating (e.g. Schlegel and St-Maurice, 1981). Theoretical calculations by St-Maurice et al. (1981) suggest that ion turbulent heating can attain a maximum of $50 \%$ of the frictional heating rate although the authors demonstrated that such heating would peak at an altitude of less than $100 \mathrm{~km}$.

If the difference between the observed and expected field-parallel ion temperature enhancements is attributed to the effect of a neutral wind in the same direction as the ion motion, a first order estimate of the neutral velocity can be calculated, using a rearranged form of Eq. (10). The assumption that the ion and neutral flows are in the same direction implies that the ion drag term constitutes the dominant term in the neutral momentum balance equation. The time constant for the response of the neutral atmosphere to ion drag is given by the neutral-ion collision frequency, which is inversely proportional to the plasma density (Baron and Wand, 1983). Electron densities observed by EISCAT during this interval at E-region altitudes are typically $1.0 \times 10^{11} \mathrm{~m}^{-3}$, yielding a response time constant of only $6 \mathrm{~h}$. The relatively high E-region electron density during this interval suggests that ion drag will constitute a significant force on the thermosphere not only in the F-region but also at E-region altitudes. If, in fact, the neutral wind is not in the same direction as the ion flow, due to the significance of other terms in the neutral momentum balance equation such as the Coriolis force, this assumption would produce the minimum value of the neutral wind magnitude required to reduce the predicted field-parallel ion temperature enhancement to that observed.

Figure 5 presents time series of the derived neutral wind velocity, with associated errors, at 125, 117 and $109 \mathrm{~km}$ altitude from 10:00 to 15:00 UT on 03 April 1992. Positive velocities indicate those where the neutral velocity is in the same sense as the ion velocity at that altitude. Where the predicted value of the field-parallel ion temperature does not exceed that observed by EISCAT, the value of the neutral wind is negative, implying motion of the neutral atmosphere is an opposite direction to that of the ion flow. The derived neutral wind at all altitudes tends to increase with time, in a manner which is consistent with the effect of ion drag over a long period of frictional heating. Furthermore, the neutral wind tends to decrease with decreasing altitude between 125 and $109 \mathrm{~km}$ altitude, also consistent with the response of the neutrals to ion drag, the time constant of which is inversely proportional to the plasma density which tends to decrease with altitude within that altitude range. 


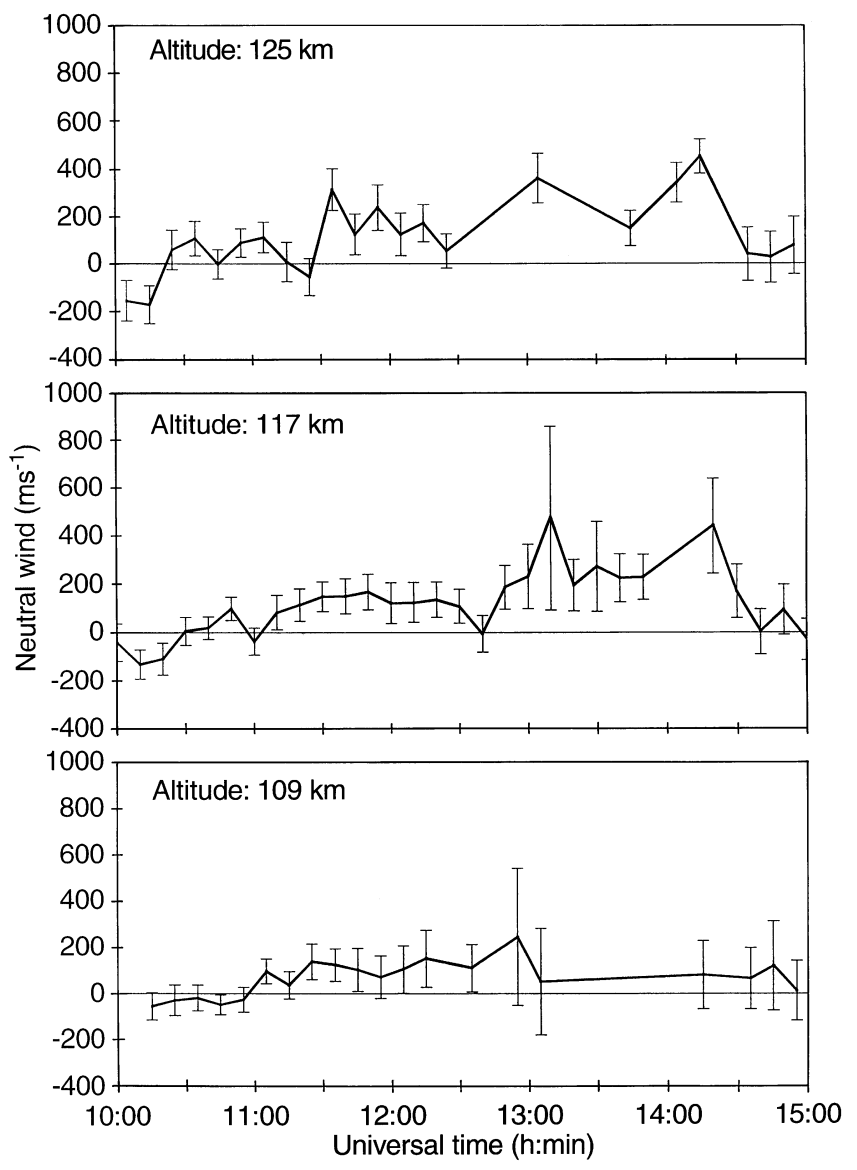

Fig. 5. E-region neutral wind velocity estimates at 125 (upper panel), 117 (middle panel) and $109 \mathrm{~km}$ altitude (lower panel), derived from EISCAT observations between 10:00 to 15:00 UT on 03 April 1992. Positive velocities indicate neutral flow in the direction of ion flow, negative velocities indicate anti-parallel ion and neutral flows

Estimates of the E-region neutral wind prior to the onset of large electric fields are not inconsistent with those determined by previous authors under quiet conditions. For example, Kunitake and Schlegel (1991) deduced equatorward neutral winds of around $80 \mathrm{~m} \mathrm{~s}^{-1}$ at $109 \mathrm{~km}$ at around 10:00 UT; at this altitude the results presented here illustrate southward neutral flow (ion flow is northward) of similar magnitude. The neutral velocity appears to decrease abruptly (over a time-scale of the order of tens of minutes) when the ions slow down, particularly at 117 and $125 \mathrm{~km}$; such as rapid deceleration of the thermosphere is unphysical, particularly given that the time constant of the neutral atmosphere is so long. It seems reasonable to speculate that, after around 14:20 UT, the neutral velocity must be underestimated. A number of factors could contribute to this effect. For example, an increase in the equilibrium ion temperature through the frictional heating event, resulting from Joule heating of the neutral atmosphere, would cause an underestimate in the neutral wind which would be more pronounced at the end of the interval when the electric field is of comparatively low value. Other terms in the ion energy balance equation may also become important such that Eq. (10) becomes less applicable.
A thorough comparison of the neutral wind with values quoted by previous authors is not appropriate, as the neutral winds are assumed parallel to the ion flow; rather, first-order estimates of the neutral wind are deduced in order to correct the values of the normalised ion-neutral collision frequency derived in the previous section.

\section{Revised estimate of the normalised collision frequency}

For such a prolonged interval of enhanced ion velocity on the dayside as observed post-noon on 03 April 1995, the neutral wind becomes significant. For less extended intervals of enhanced ion flow or those on the nightside where the electron density is lower, ion drag would have a far less pronounced effect on the lower thermosphere. The results highlighted in the previous section indicate that the assumption adopted in the calculation of the normalised collision frequency, that the neutral wind is negligible, is not necessarily valid under all circumstances. Expressions (7) and (8) were employed in Sect. 4 to derive two independent estimates of the ratio of the ionneutral collision frequency to the ion gyrofrequency from the rotation of the ion velocity vector and reduction in the velocity magnitude from that observed in the F-region. In the present section, the normalised collision frequency is calculated by Eq. (4) and (5), incorporating the E-region neutral winds derived in the previous section. The calculated neutral winds derived in the E-region are themselves first-order estimates, assuming neutral flows parallel to the direction of the ion velocity. Moreover, the calculation of the neutral wind, or more specifically the field-parallel ion temperature partition coefficient and the mean neutral mass, itself requires modelled values of the ion-neutral collision frequency. This introduces an inconsistency, as the neutral wind is subsequently being used to derive corrected values of the normalised collision frequency. The inclusion of the neutral wind is, however, not intended to provide definitive results, rather to gauge a general effect of a neutral wind on calculations of the normalised collision frequency by this method.

Figure 6 presents a time series of the revised estimate of the normalised ion-neutral collision frequency at 125 (upper panel), 117 (central panel) and $109 \mathrm{~km}$ (lower panel) altitude from 10:00 to 15:00 UT on 03 April 1992 , derived from the rotation of the relative ionneutral velocity vector from the direction of $\mathbf{E}_{\mathrm{eff}} \times \mathbf{B}$ (solid line). For comparison, the estimate of the normalised collision frequency calculated from the rotation of the ion velocity vector from the $\mathbf{E} \times \mathbf{B}$ direction is included (dashed line) as are the modelled values (dotted line). As the neutral gas is assumed to flow in the direction of the E-region ions, the direction of relative ion-neutral flow will be the same as that of the observed ion velocity; the effective electric field will, however, be oriented in a different direction to the imposed electric field. Figure 7 illustrates a corresponding time series of the revised estimates of the normalised 

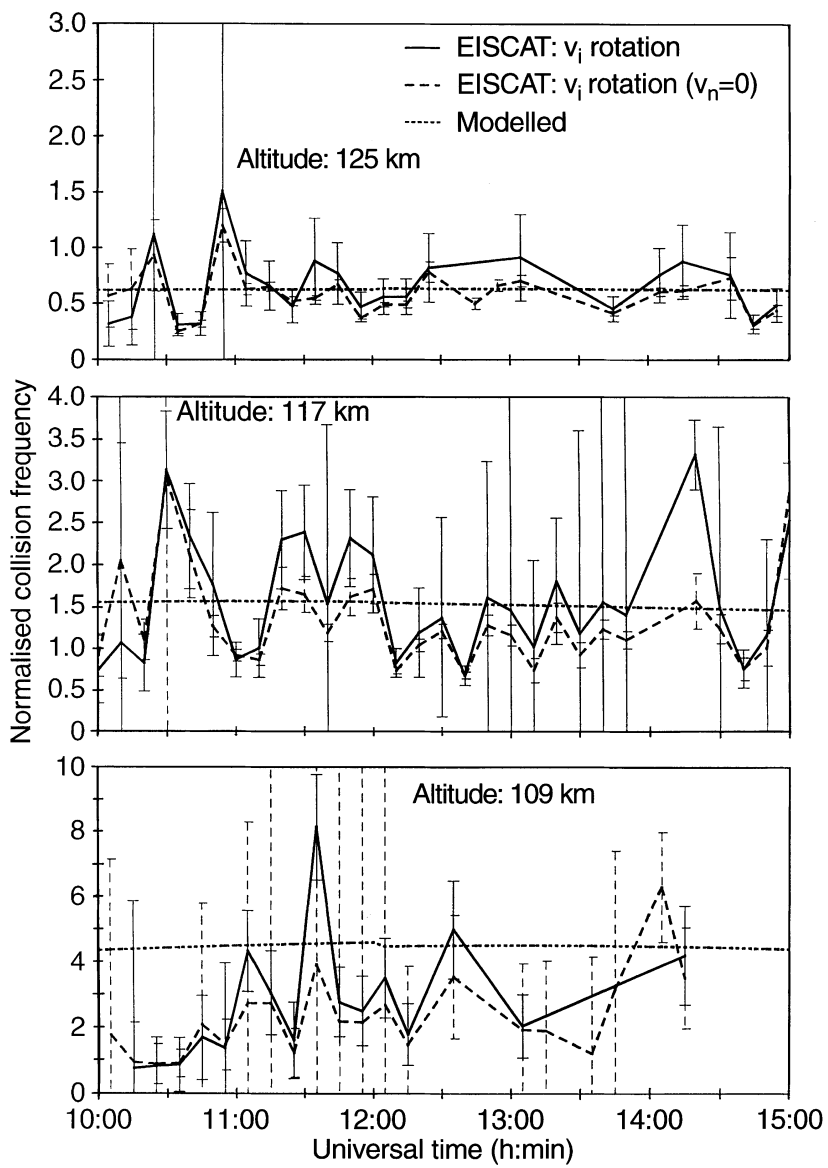

Fig. 6. Normalised ion-neutral collision frequency derived from EISCAT observations of ion velocity rotation including a neutral wind correction (full line), at 125 (upper panel), 117 (middle panel) and $109 \mathrm{~km}$ altitude (lower panel). These derived neglecting the neutral wind (as shown in Fig. 3) are illustrated, for comparison, as a dashed line and modelled values are represented by a dotted line

ion-neutral collision frequency derived from the reduction in magnitude of the relative ion-neutral velocity from that of $\mathbf{E}_{\mathrm{eff}} \times \mathbf{B}$ (solid line). Again, values of the normalised collision frequency derived assuming a stationary thermosphere and modelled values are also included, represented by dashed and dotted lines, respectively. The mean and standard deviation of normalised collision frequency, calculated between 11:00 and 14:30 UT, derived from EISCAT observations of the rotation in the ion-neutral difference velocity and reduction in its magnitude are presented in Table 2.

At all altitudes, the normalised ion-neutral collision frequency derived with the inclusion of a first-order estimate of the neutral wind tends to be greater than that derived ignoring thermospheric flow. It is noticeable, however, that the converse tends to be true when the neutral flow is oppositely directed to that of the ions. Although the inclusion of a neutral wind does tend to increase the estimates of the normalised collision frequency towards the modelled values, the difference in the derived values of the normalised ion-neutral collision frequency introduced with the inclusion of a non-zero neutral wind is generally less than the estimated error in the normalised collision frequency.


Fig. 7. Normalised ion-neutral collision frequency derived from EISCAT observations of ion velocity magnitude reduction including a neutral wind correction (full line), at 125 (upper panel), 117 (middle panel) and $109 \mathrm{~km}$ altitude (lower panel). Those derived neglecting the neutral wind (as shown in Fig. 3) are illustrated, for comparison, as a dashed line and modelled values are represented by a dotted line

The gradual increase in the normalised collision frequency at $109 \mathrm{~km}$, as noted in a previous section, is still apparent even after the neutral wind correction. Indeed, the collision frequency at this altitude more than doubles over the $5 \mathrm{~h}$ interval. EISCAT observations, presented by Flå et al. (1985), revealed an increase in the ion-neutral collision frequency, between 90 and $105 \mathrm{~km}$ altitude, by a factor of $1.5-2$ over a 90 min interval. Flå et al. (1985) speculated that this was due to neutral

Table 2. Mean normalised ion-neutral collision frequencies derived from EISCAT observations taken between 11:00 and 14:30 UT, taking into consideration the effect of neutral motion. Standard deviations are also given

\begin{tabular}{|c|c|c|c|c|}
\hline \multicolumn{5}{|c|}{ Normalised ion-neutral collision frequency } \\
\hline \multirow{2}{*}{$\begin{array}{l}\text { Altitude } \\
(\mathrm{km})\end{array}$} & \multicolumn{2}{|c|}{ Velocity rotation } & \multicolumn{2}{|c|}{ Velocity reduction } \\
\hline & mean & standard deviation & mean & standard deviation \\
\hline 125 & 0.7 & 0.2 & 0.7 & 0.3 \\
\hline 117 & 1.6 & 0.7 & 1.3 & 0.5 \\
\hline 109 & 3.3 & 1.2 & 4.9 & 0.9 \\
\hline
\end{tabular}


density enhancements, caused by atmospheric heating in response to the passage of a large-amplitude, longperiod gravity wave; particle, Joule and wave turbulent heat sources were discounted by the authors as, during their interval, only a moderate electric field (around $20 \mathrm{mV} \mathrm{m}^{-1}$ ) was present. During the present more prolonged interval, with an electric field exceeding $100 \mathrm{mV} \mathrm{m}^{-1}$ at maximum, Joule and wave heating (there is no evidence of particle precipitation) could well account for the neutral density, and hence collision frequency, increase at $109 \mathrm{~km}$. Indeed, Flå et al. (1985) state that it is around $110 \mathrm{~km}$ that these geophysical heating mechanisms will have most effect.

The neutral wind correction has generated a number of prominent spikes in the time series of normalised ionneutral collision frequency, especially at the lower altitudes of 117 and $109 \mathrm{~km}$; such spikes are unlikely in a slowly varying quantity like collision frequency. This indicates that, under some circumstances, the inclusion of the neutral wind estimate can induce error, rather than correct it. However, whilst the estimation of neutral wind, which includes several assumptions, may not be particularly accurate, its inclusion provides a valuable indicator of the general effect of thermospheric motion on normalised collision frequencies derived by this method, especially for such a protracted event in which significant neutral winds can be induced.

Overall, the inclusion of the derived neutral wind term does not appear to have a significant effect on the values of the normalised ion-neutral collision frequency, although the difference between the results does relate to the effect of assuming that the neutral wind is in the same direction as the ion flow.

The way in which the present authors have attempted to account for neutral winds is very different to that adopted by Nygrén et al. (1989). As noted previously, Nygrén et al. (1987) suggested an approach by which their method for the determination of the ion-neutral collision frequency could be amended to overcome the limitation of neglecting atmospheric motion; a detailed explanation of the modified method including its limitations, along with experimental results, were subsequently presented by Nygrén et al. (1989). Basically, use of both vertical and eastward-tilted profiles of ion velocity enabled Nygrén et al. (1989) to eliminate the horizontal component of the neutral wind from their expression for the normalised ion-neutral collision frequency, without the need for an independent measure of neutral wind velocities as is more the case in the present method. The results of Nygrén et al. (1989) indicated that although the effect of the neutral wind could be small, this was not necessarily the case. Indeed, under some circumstances, the authors found that neglecting the neutral wind, yielded results which were not sensible.

\section{Summary and conclusions}

EISCAT CP-1-J observations on 03 April 1992, revealed an extended interval during which the perpendicular electric field was significantly enhanced, to values in excess of $100 \mathrm{mV} \mathrm{m}^{-1}$. From both the rotation of the ion velocity vector and reduction in the velocity magnitude with decreasing altitude during the interval, estimates of the ratio of the ion-neutral collision frequency to ion gyrofrequency were made at the CP1-J E-region tristatic altitudes, which were compared to predictions based on the MSIS- 86 thermospheric model and IGRF model of the geomagnetic field. The discrepancy between the modelled normalised collision frequency and that derived from the EISCAT observations a lower E-region altitudes is attributed to an overestimate of the neutral densities produced by MSIS-86, in accord with previous authors.

Furthermore, from the ion velocity measurements during this interval, the extent of frictional heating of the ion population at the E-region tristatic altitudes was derived from a simplified form of the ion energy balance equation. The predicted enhancement in the field-parallel ion temperature was compared to those observed, and, by attributing the discrepancy to thermospheric motion in response to an ion drag force on the neutral atmosphere, first-order estimates of the neutral wind velocity were obtained. These thermospheric flows, the values of which are not inconsistent with previous measurements, were further employed to derive revised estimates of the normalised collision frequency. Their inclusion, however, is not particularly significant at the altitudes under consideration, although it does tend to push the derived values of the normalised collision frequency nearer to those modelled.

If this, and previous, methods for the determination of the ion-neutral collision frequency could be coordinated into a large-scale project, employing extensive data sets, the value of both EISCAT and the new EISCAT Svalbard radar is upper atmosphere studies would be enhanced such that future versions of atmospheric models could have a strong contribution from these radars.

Acknowledgements. Thanks are due to the director of EISCAT and his staff for operating the facility and supplying the data. EISCAT is an association of the national scientific agencies of Finland, France, Germany, Japan, Norway, Sweden and the United Kingdom. J. A. Davies is supported by a grant from the Particle Physics and Astronomy Research Council.

Topical Editor D. Alcaydé thanks T. Nygrén and another referee for their help in evaluating this paper.

\section{References}

Baron, M. J., and R. H. Wand, F-region ion temperature enhancements resulting from Joule heating, J. Geophys. Res., 88, 4114, 1983.

Brekke, A., R. J. Doupnik, and P. M. Banks, A preliminary study of the neutral wind in the auroral E-region, J. Geophys. Res., 78, 8235, 1973.

Brekke, A., C. Hall, and Ø. Pettersen, EISCAT UHF studies of ionosphere currents on June 16-17, 1987, Ann. Geophysicae, 8, 213, 1990.

Brekke, A., S. Nozawa, and T. Sparr, Studies of the E-region neutral wind in the quiet auroral ionosphere, J. Geophys. Res., 99, 8801, 1994. 
Davies, J. A., M. Lester, B. Jenkins, and R. J. Moffett, Dayside ion frictional heating: EISCAT observations and comparison with model results, J. Atmos. Terr. Phys., 57, 775, 1995.

Du Castel, F., and G. Vasseur, Evaluation des performances d'un sondeur ionosphérique à diffusion incohérent, Ann. Telecommun., 27, 239, 1972.

Flå, T., S. Kirkwood, and K. Schlegel, Collision frequency measurements in the high-latitude E-region with EISCAT, Radio Sci., 20, 785, 1985.

Hagfors, T., and R. A. Brokelman, A theory of collision dominated fluctuations in a plasma with applications to incoherent scattering, Phys. Fluids, 14, 1143, 1971.

Hedin, A. E., MSIS-86 thermospheric model, J. Geophys. Res., 92, 4649, 1987.

Huuskonen, A., High-resolution studies of the lower E-region using the incoherent scatter method, $\mathrm{PhD}$ Thesis, University of Oulu, 1988.

Huuskonen, A., High-resolution observations of the collision frequency and temperatures with the EISCAT UHF radar, Planet. Space Sci., 37, 211, 1989.

Huuskonen, A., T. Nygrén, L. Jalonen, and J. Oksman, The effect of electric field-induced vertical convection on the precipitation Elayer, J. Atmos. Terr. Phys., 46, 927, 1984.

Huuskonen, A., T. Nygrén, L. Jalonen, T. Turunen, and J. Silen, High resolution EISCAT observations of the ion-neutral collision frequency in the lower E-region, J. Atmos. Terr. Phys., 48, 827, 1986.

Kirkwood, S., Seasonal and tidal variations of neutral temperatures and densities in the high-latitude lower thermosphere measured by EISCAT, J. Atmos. Terr. Phys., 48, 817, 1986.

Kofman, W., C. Lathuillère, and B. Pibaret, Neutral atmosphere studies in the altitude range $90-110 \mathrm{~km}$ using EISCAT, $J$. Atmos. Terr. Phys., 48, 837, 1986.

Kunitake, M., and K. Schlegel, Neutral winds in the lower thermosphere at high latitudes from five years of EISCAT data, Ann. Geophysicae, 9, 143, 1991

Lathuillère, C., V. B. Wickwar, and W. Kofman, Incoherent scatter measurements of ion-neutral collision frequencies and temperatures in the lower thermosphere of the auroral region, $J$. Geophys. Res., 88, 137, 1983.

Lathuillère, C., D. Hubert, C. La Hoz, and W. Kofman, Evidence of anisotropic temperatures of molecular ions in the auroral ionosphere, Geophys. Res. Lett., 18, 163, 1991.

Løvhaug, U. P., and T. Flå, Ion temperature anisotropy in the auroral F-region as measured with EISCAT, J. Atmos. Terr. Phys., 48, 959, 1986.

McBride, J. B., E. Ott, J. P. Boris, and J. H. Orens, Theory and simulation of turbulent heating by the modified two-stream instability, Phys. Fluids, 15, 2367, 1972.

McCrea, I. W., M. Lester, T. R. Robinson, N. M. Wade, and T. B. Jones, On the identification and occurrence of ion frictional heating events in the high-latitude ionosphere, J. Atmos. Terr. Phys., 53, 587, 1991.

McCrea, I. W., M. Lester, T. R. Robinson, J.-P. St-Maurice, N. M. Wade, and T. B. Jones, Derivation of the ion temperature partition coefficient $\beta_{\|}$from the study of ion frictional heating events, J. Geophys. Res., 98, 15701, 1993.
McCrea, I. W., G. O. L. Jones, and M. Lester, The BEAN experiment - an EISCAT study of ion temperature anisotropies, Ann. Geophysicae, 13, 177, 1995.

Nygrén, T., Studies of the E-region ion neutral collision frequency using the EISCAT incoherent scatter radar, Adv. Space Res., 18, 79, 1996.

Nygrén, T., L. Jalonen, J. Oksman, and T. Turunen, The role of electric field and neutral wind direction in the formation of sporadic E-layers, J. Atmos. Terr. Phys., 46, 373, 1984.

Nygrén, T., L. Jalonen, and A. Huuskonen, A new method of measuring the ion-neutral collision frequency using incoherent scatter radar, Planet. Space Sci., 35, 337, 1987.

Nygrén, T., B. S. Lanchester, L. Jalonen, and A. Huuskonen, A method for determining ion-neutral collision frequency using radar measurements of ion velocity in two directions, Planet. Space Sci., 37, 494, 1989.

Salah, J. E., J. V. Evans, and R. H. Wand, E-region measurements at Millstone Hill, J. Atmos. Terr. Phys., 37, 461, 1975.

Schlegel, K., and J.-P. St-Maurice, Anomalous heating of the polar E-region by unstable plasma waves: 1 . Observations, $J$. Geophys. Res., 86, 1447, 1981

Schlegel, K., H. Kohl, and K. Rinnert, Temperature and collision frequency in the polar-E region measured with the incoherent scatter technique, J. Geophys. Res., 85, 710, 1980.

Schunk, R. W., and A. F. Nagy, Ionospheres of terrestrial planets, Rev. Geophys. Space Phys., 18, 813, 1980.

Schunk, R. W., and J. C. G. Walker, Ion velocity distributions in the auroral ionosphere, Planet. Space Sci., 20, 2175, 1972.

Schunk, R. W., and J. C. G. Walker, Theoretical ion densities in the lower ionosphere, Planet. Space Sci., 21, 1875, 1973.

St-Maurice, J.-P., and R. W. Schunk, Auroral ion velocity distributions for a polarization collision model, Planet. Space Sci., 25, 243, 1977.

St-Maurice, J.-P., K. Schlegel, and P. M. Banks, Anomalous heating of the polar E-region by unstable plasma waves: 2 . Theory, J. Geophys. Res., 86, 1453, 1981.

Virdi, T. S., and P. J. S. Williams, Altitude variations in the amplitude and phase of tidal oscillations at high-latitude, $J$. Atmos. Terr. Phys., 55, 697, 1993.

Virdi, T. S., G. O. L. Jones, and P. J. S. Williams, EISCAT observations of the E-region semidiurnal tide, Nature, 324, 354, 1986.

Wand, R. H., Semidiurnal tide in the E-region from incoherent scatter measurements at Arecibo, Radio Sci., 11, 641, 1976.

Wand, R. H., and F. H. Perkins, Radar Thomson scatter observations of temperature and ion-neutral collision frequency in the E-region, J. Geophys. Res., 78, 6370, 1968.

Wickwar, V. B., C. Lathuillère, W. Kofman, and G. Lejeune, Elevated electron temperatures in the auroral E-layer measured with the Chatanika radar, J. Geophys. Res., 86, 4721, 1981.

Williams, P. J. S., and T. S. Virdi, EISCAT observations of tidal modes in the lower ionosphere, J. Atmos. Terr. Phys., 51, 569, 1989.

Williams, P. J. S., T. S. Virdi, S. W. H. Cowley, and M. Lester, Short-lived bursts of plasma velocity in the auroral zone: 1 . Observational evidence from radar measurements. J. Atmos. Terr. Phys., 52, 421, 1990. 